\title{
La construcción del bielsismo en el diario La Nación
}

\section{The construction of bielsismo in the newspaper La Nación}

\author{
Gabriel Enrique Irungaray girungaray@yahoo.com \\ https://orcid.org/0000-0001-7440-6001
}

Facultad de Periodismo y Comunicación Social; Universidad Nacional de La Plata (Argentina)

\section{Resumen}

El bielsismo es una construcción discursiva que dialoga y se tensiona con un contexto formado por enunciados, mitos y tradiciones del fútbol nacional. Para indagar en esos discursos será necesario analizar un corpus de 6 notas del año 2019, donde Bielsa se encuentra dirigiendo el Leeds United Fútbol Club. El corte sincrónico tiene la intención de analizar la primera temporada del técnico en el equipo inglés, con el que logró asentarse en los primeros puestos durante la mayor parte del torneo, pero que finalmente no logró el ascenso a la primera división británica, la Premier League. 
Palabras Clave: Bielsa; fútbol; La Nación; análisis del discurso

Abstract

The bielsismo is a discursive construction that dialogues and is stressed with a context formed by statements, myths and traditions of national football. To investigate these speeches it will be necessary to analyze a corpus of 6 notes of the year 2019, where Bielsa is directing the Leeds United Football Club. The synchronous court intends to analyze the first season of the coach in the English team, with which he managed to settle in the top positions during most of the tournament, but finally failed to promote the first British division, the Premier League.

Keywords: Bielsa; football; La Nación; analysis of discourse

La construcción mediática de Marcelo Bielsa creó un relato unificado y complejo que lo representa hegemónica y discursivamente. Los conceptos de victoria y derrota en los discursos del fútbol argentino, el legado deportivo de la Selección Argentina, la mediatización y mercantilización del deporte, y la propia persona de Bielsa, son representaciones que el diario La Nación utilizó para construir la figura del entrenador. Será importante tener en cuenta qué construcción de bielsismo realiza el medio para tomar una muestra cualitativa de los diversos discursos que componen el imaginario sobre el entrenador. El presente análisis tiene como objetivo agregar nuevas reflexiones al Trabajo Integrador Final denominado «El Gráfico: épica, potrero y bielsismo en el relato del fútbol argentino». ${ }^{1}$

\section{El análisis de discurso como metodología}

Para hablar de relatos es necesario referir qué son los discursos sociales. Atendiendo la definición de Marc Angenot (2010), el discurso social es todo lo que se imprime, se habla, se narra y se argumenta en un estado de sociedad. Sin embargo, hay discursos que se imponen

\footnotetext{
${ }^{1}$ Título del Trabajo Integrador Final de grado del autor de este artículo. 
por sobre otros a partir de reglas prescriptivas que los integran y cohesionan, obedeciendo a esquematizaciones de lo decible y lo escribible (Angenot, 2010).

Por otro lado es necesario reconocer que el acto de enunciación responde a la utilización de los usos lingüísticos, partes primarias que conforman los enunciados y que funcionan en interdependencia con el contexto, que es el marco desde se eligen esos enunciados (Calsamiglia y Tusón, 2001). Es lo que Dominique Maingueneau (2009) define como interdiscurso, referido a la interacción con los enunciados que nos rodean históricamente y en diferentes situaciones.

La elección de los usos lingüísticos que utilice La Nación en su producción enunciativa será capital para analizar sus intenciones y estrategias comunicativas sobre Marcelo Bielsa. En este caso, el análisis estará orientado a encontrar esas regularidades y previsibilidades (Angenot, 2010) que construyan la figura del entrenador. Dentro de esta heterogeneidad de discursos que involucran deporte, medios de comunicación, mitos y tradiciones nacionales, se refractarán ${ }^{2}$ diversos géneros discursivos que se articulan y se tensionan. Todas estas esferas de la actividad de la lengua son tan variadas e inagotables como las prácticas de la actividad humana (Bajtín, 1982). Es por eso que los discursos sobre Bielsa se conformarán de enunciados heterogéneos pero confluyentes, en tanto y en cuanto ejecutan discursos esquematizados y hegemónicos ${ }^{3}$ sobre su persona.

Para realizar un análisis de los enunciados y la manera en que se enuncian, se debe reconocer a la lengua como algo dinámico y cambiante, un objeto viviente que es transformado pero que también transforma. Dentro de cada enunciado hay multiplicidad de puntos de vista y lenguajes (Calsamiglia y Tusón, 2001), distintas voces que lo conforman de manera simultánea. Es la definición que Bajtín utiliza para hablar de polifonía, comprendiéndolo como un plurilingüismo compuesto de visiones del mundo, lenguajes y voces sociales de diversas autorías a través del dialogismo (Puig, 2004). En el presente análisis, los elementos polifónicos serán analizados a partir del sujeto hablante y teniendo en cuenta 3 entidades polifónicas: el sujeto empírico como productor del enunciado; el locutor, como responsable del enunciado y la enunciación (y que usualmente se presenta en primera persona); y los enunciadores, que son las otras voces que aparecen en el discurso (Calsamiglia y Tusón, 2001; Secul Giusti, 2016).

\footnotetext{
${ }^{2}$ La refracción es un concepto utilizado por Valentín Voloshinov y refiere a la distorsión de la realidad filtrada por nuestras ideas a través de la mediación del signo.

${ }^{3}$ El concepto de hegemonía del discurso parte de Angenot, entendido como un conjunto complejos de reglas prescriptivas de lo decible, cohesionadas e integradas. El discurso es un objeto compuesto a partir de ellas. 
Por otro lado, las modalidades son los elementos que permiten dar cuenta de la subjetividad del hablante en sus enunciados porque en ellas participa con sus perspectivas y actitudes en la interacción comunicativa. Para este análisis será necesario atender tanto a la modalidad de enunciado como a la de enunciación.

Las modalidades de enunciación que utilizaré serán asertivas, donde el hablante presenta su enunciado como algo cierto; y exclamativas, las cuales indican una expresión enfática. Respecto de las modalidades de enunciado, se encuentran las lógicas, que sitúan el enunciado en relación con la certeza; y las apreciativas, que se relacionan con lo feliz o lo triste, y con categorías apreciativas como lo malo, lo bueno y lo peyorativo (Calsamiglia y Tusón, 2001; Secul Giusti, 2016).

Finalmente, será necesario analizar los elementos deícticos que se encuentran dentro del corpus para dar cuenta de su relación directa con el contexto. Esto quiere decir que su significado concreto depende de la situación de enunciación tomando en consideración quién las pronuncia, a quién, cuándo y dónde. Dentro de las referencias deícticas será necesario utilizar las personales (yo, tú, nosotros), que pueden hallarse en los locutores o sujetos empíricos de la situación enunciativa (Calsamiglia y Tusón, 2001).

Estas herramientas serán útiles para dar cuenta de las regularidades que construyen el bielsismo, referentes tanto al estilo de juego del entrenador como a la construcción mediática por fuera del campo de juego, que se vuelve mercancía a partir de su mediatización (Irungaray, 2019).

\section{Fútbol y nación en la matriz discursiva del bielsismo}

Comprender la performatividad de los discursos de la nación exige tener en cuenta dos aspectos fundamentales. El primero de ellos es la dicotomía central del pensamiento nacional creada por Domingo Sarmiento en 1845, denominada civilización o barbarie. El segundo obedece al año 1880, y conjuga el triunfo del ideario liberal como proyecto político con la proyección discursiva, territorial y cultural de la «Generación del '37». La «razón occidental» imponía condiciones y se proyectaba como la opción dicotómica de los incivilizados o bárbaros, acallados por el triunfo de las ideas europeas en el territorio (Moyano, 2009). La alteridad será un rasgo discursivo fuerte en la esfera política y cultural, y decantará años después dentro de la esfera del deporte. Esto conformará una relación inextricable: los relatos performativos del fútbol se articularán con los discursos de la nación.

A partir de esta afirmación es necesario describir cuales son los dos momentos centrales en la conformación del fútbol criollo. El primero de ellos ocurrirá en 1880, con la llegada de obreros 
ingleses que poco a poco irán introduciendo la práctica del fútbol en el país; el segundo es en 1914, cuando Racing Club de Avellaneda gana el primer campeonato local sin ningún jugador británico en su plantel (Archetti, 2008).

Este momento fundacional del fútbol local será denominado por Pablo Alabarces (2002) como criollización, definiendo de esa manera la reconversión del fútbol británico como una de las características del nacionalismo deportivo. La alteridad nosotros/ellos para diferenciar el fútbol local del británico, y el soporte sobre las primeras victorias internacionales de equipos argentinos y de la propia Selección, son otras características que el autor cita para referirse a la relación articulada entre nación y fútbol. ${ }^{4}$ Esto último es importante porque será la marca de fuego de los discursos sobre el fútbol local referidos a la victoria y la derrota.

Ganar y perder son dos hechos que están estrechamente ligados al bielsismo. Por un lado, porque en un deporte de competencia como el fútbol ambos resultados conviven con la disciplina. Por el otro, porque el legado deportivo de Marcelo Bielsa está articulado directamente al Mundial de Corea-Japón 2002 y la eliminación en primera ronda de la Selección Argentina ${ }^{5}$, algo que el entrenador denominó como fracaso:

\begin{abstract}
Dispongo de la fuerza, la entereza y la disposición para intentarlo nuevamente. Más allá del fracaso en el Mundial, tiene valor lo hecho anteriormente por el equipo, aunque no se haya cristalizado luego del modo esperado. Me sorprendió que me ofrecieran continuar, porque no es frecuente que haya continuidad después de un fracaso, pero no necesito revancha. Yo jamás utilice esa palabra (lucht, 2011, p. 297).
\end{abstract}

Este legado será importante porque guarda importancia en el imaginario nacional a través de su espesor histórico. Esto significa que el bielsismo no solamente dialoga con la alteridad nacional y la performatividad de la victoria y la derrota, sino también con otros enunciados mitológicos y tradicionales. Como define Archetti (2008), una tradición futbolística se construye no sólo a partir de una continuidad histórica, sino que su esencia adquiere espesor a partir de los triunfos. Los enunciados de La Nación tendrán un diálogo constante con estos discursos.

\footnotetext{
${ }^{4}$ La cuarta característica a la que se refiere Alabarces es el soporte en héroes locales. En los primeros años de la década del '20, ese lugar comenzó a ser ocupado por futbolistas y otros deportistas, sobre todo a partir de las caracterizaciones de la naciente revista El Gráfico.

${ }^{5}$ El legado de la Selección Argentina (2 Copas del Mundo y 14 Copa América), articulados a la Eliminatoria previa al Mundial 2002 ganada por la Selección Nacional, se presentaban como dos elementos contundentes que legitimaban un posible éxito del conjunto argentino en aquella competencia.

Question, Vol. 1, N. ${ }^{\circ}$ 65, abril 2020. ISSN 1669-6581

Instituto de Investigaciones en Comunicación | Facultad de Periodismo y Comunicación Social | Universidad Nacional de La Plata 
Análisis del bielsismo: estilo de juego, legado deportivo y fútbol espectáculo

Marcelo Bielsa fue presentado como director técnico del Leeds United Fútbol Club el 25 de junio de 2018. Las notas elegidas para el análisis toman como referencia la primera temporada del equipo disputando el Championship, torneo de la segunda división de Inglaterra. Esa primera temporada de Bielsa estará surcada por 3 hechos paradójicos y particulares, características que componen las estrategias enunciativas de La Nación.

El primero de ellos será el denominado Spygate ${ }^{6}$, el cual puso en primera plana de la prensa al Leeds dejando de lado los resultados deportivos del equipo, en ese momento puntero del Championship. Otro hecho paradigmático se dio en el último partido del torneo donde el Leeds convirtió un gol con un jugador rival del Aston Villa lesionado en el campo de juego, y que derivó en una orden de Bielsa para ceder un gol al rival. El último hecho se relaciona con la chance de ascender del Leeds, que se mantuvo en los primeros puestos durante la mayor parte del torneo, pero que sin embargo no pudo ascender directamente ni tampoco clasificar desde los play offs para jugar la final por el tercer ascenso.

Las notas del diario La Nación articulan este proceso deportivo de Bielsa con las concepciones de victoria y derrota del nacionalismo deportivo, la caracterización de la locura, el fútbol espectáculo y el legado deportivo que implica el estilo del entrenador.

En la nota «Bielsa y un clásico impensado por obra de viejas glorias del Manchester United» (La Nación, 2019, p. 1), se hace referencia a un hecho imprevisto. El sujeto empírico posiciona primero a Bielsa y crea la situación de enunciación a partir de la sorpresa y la casualidad. Estos aspectos difieren del estilo del entrenador, que busca la minimización del error y tiene como predilección la pasión por el detalle. El conocimiento pormenorizado del rival, la disciplina táctica y la importancia de los entrenamientos se aúnan en la idea de orden, disciplina y ataque, y la estrategia enunciativa del medio se pone en tensión con estas características, una marca fundamental del bielsismo.

Por otro lado, el enunciado «viejas glorias del Manchester United» (La Nación, 2019, p.1) propone dos enunciadores polifónicos distintos, ambos históricos. El primero de ellos porque reaviva la rivalidad deportiva entre Leeds United y Manchester United, ${ }^{7}$ que decanta en el fútbol

${ }^{6}$ Se denominó así a la polémica generada luego de que un asistente de Bielsa fuera descubierto espiando un entrenamiento del Derby County, equipo que competía con el Leeds en el Championship. En la serie de 6 capítulos sobre la temporada del equipo en el torneo, denominado «Take us home: Leeds United», el episodio 3 está dedicado a aquel hecho.

7 De acuerdo a la información de la página oficial del Manchester United, la rivalidad de Leeds y Manchester United data del siglo XV, donde se dio la denominada «Guerra de las Rosas» entre la Casa 
por una histórica guerra civil por la corona de Inglaterra. El otro, porque expone y dialoga con el interdiscurso poniendo de manifiesto que ex jugadores victoriosos del Manchester United se enfrentarán a Bielsa. ${ }^{8}$ En el cuerpo de la nota, La Nación describe:

«En la temporada pasada, el equipo del Loco tuvo un balance negativo en las series eliminatorias: disputó cuatro y perdió tres» (La Nación, 2019. p. 1).

Se modaliza el enunciado «balance negativo" de manera lógica a partir de los resultados de Bielsa en partidos de eliminación. También se articulan los enunciados del nacionalismo deportivo referidos a la victoria y la derrota, performativos del relato fundacional del fútbol argentino que se gesta por el subcampeonato de la Selección Argentina en el Mundial de Uruguay 1930, la gira internacional de Boca Juniors en 1925 y la medalla de plata en las Olimpiadas de Ámsterdam en 1928 (Alabarces, 2002; Archetti, 2008).

Por otro lado, el entrenador aparece caracterizado a partir de la palabra loco. Desde la época clásica, la locura ha sido identificada desde la exclusión, la segregación y el miedo, formas de exclusión social que funcionan estructuralmente. Los primeros que cargaron con estos sentidos fueron los leprosos, alejados de las ciudades y confinados en una colonia en cuarentena (Foucault, 1964). En este caso, el enunciado «el equipo del Loco» (La Nación, 2019, p. 1) modaliza de manera lógica la certeza de que se trata del equipo de Marcelo Bielsa a partir de esquematizaciones sobre la figura del entrenador que naturalizan la palabra loco para referirse a su persona.

Luego la nota ofrece más datos:

Dos fechas después del comienzo del Championship, competencia que para Leeds es prioridad en la temporada porque es la que posibilita el ascenso a la Premier League, a Marcelo Bielsa el calendario le fija para este martes un partido de eliminación directa por la Copa de la Liga (Carabao Cup es el nombre comercial). De visitante, a las 15.45 de la Argentina (transmite ESPN 3), se enfrentará con Salford City, que participa en la League Two (cuarta división, dos por debajo de la de Leeds) (La Nación, 2019, p. 1).

En este enunciado se referencia el legado deportivo del entrenador en el Leeds, correspondiente a la temporada pasada, en donde no pudo conseguir el ascenso más allá de

de York y la Casa Lancaster, respectivamente. Posteriormente, en los siglos XVIII y XIX los conflictos continuaron durante la Revolución Industrial.

8 Con Ferguson, los red devils lograron ganar 12 ligas locales, 2 UEFA Champions League, 1 Copa Intercontinental, 5 FA Cup, 4 Copas de Liga, 1 Supercopa de la UEFA, 10 Community Shield y 1 Copa de la UEFA. 
haber terminado en los primeros puestos. ${ }^{9}$ La palabra prioridad funciona como una estrategia de enunciación asertiva porque ubica el campeonato por sobre otras competencias. En segundo plano, se indica que el rival del Leeds es el Salford, un dato que queda detrás del título principal y la referencia al Manchester United.

Por otro lado, se manifiesta la importancia que otorga el sujeto empírico a la estadística referida a Bielsa y los partidos de eliminación directa. El dato guarda características negativas porque refiere a derrotas del Leeds en competencias mano a mano. La articulación entre los resultados negativos y la tradición triunfalista del fútbol local se vuelve central porque esquematiza los enunciados sobre Bielsa y el Leeds.

Respecto al encuentro, el medio citará directamente la palabra de Bielsa:

\begin{abstract}
La rivalidad Leeds-Manchester es un agregado, pero para nosotros es un partido muy interesante, tenga o no vinculación con Manchester. Conozco los antecedentes, la historia del partido y la rivalidad, también la relación de Manchester United con los propietarios de Salford. Afrontaremos el partido con la máxima seriedad, como hacemos con todos. Mucho más no puedo decir, seguramente ustedes (por los periodistas) tienen más fundamentos para hablar sobre el tema, expresó Bielsa (La Nación, 2019, p. 2).
\end{abstract}

Aquí se manifiesta una opinión diferente a la del medio porque el locutor es Bielsa, que rápidamente deja en segundo plano la rivalidad con el Manchester United utilizando la palabra agregado. El adjetivo seriedad puede comprenderse desde la concentración pero también desde la responsabilidad, ambas características positivas. La precisión de Bielsa en la elección de los usos lingüísticos es un rasgo crucial para entender su relación con el periodismo. ${ }^{10}$

La estrategia del entrenador difiere de La Nación y produce una tensión discursiva cuando Bielsa pone en segundo plano la referencia y les otorga a los periodistas «más fundamentos» para hablar sobre la rivalidad con el Manchester United. Lo que el entrenador expone es una capacidad de retórica, de argumentación por parte del periodismo. Como disciplina, la retórica ha sido desarrollada por autores como Platón, quien arguyia que los sofistas eran propulsores de una retórica falsa que proponía la seducción y la persuasión por medio de la palabra,

9 La primera parte del torneo, culminada en diciembre de 2018, el Leeds United culminó en la primera posición. Con el correr de la segunda rueda, osciló entre el primer y tercer puesto. Finalmente no logró el ascenso directo y cayó en la ronda de playoffs frente al Derby County que otorgaba una tercera plaza de ascenso.

10 Durante sus conferencias de prensa como técnico de la Selección Argentina, Román lucht explica que los periodistas se iban decepcionados al no obtener declaraciones «marketineras» del entrenador. En ese sentido, lucht explica que la caracterización de los periodistas ante ese tipo de conferencia era calificar a Bielsa de aburrido y poco entendible.

Question, Vol. 1, N. ${ }^{\circ}$ 65, abril 2020. ISSN 1669-6581

Instituto de Investigaciones en Comunicación | Facultad de Periodismo y Comunicación Social | Universidad Nacional de La Plata 
dejando de lado la reflexión (García García, 2005). ${ }^{11}$ La disputa entre una retórica verdadera y otra verosímil forma parte del enunciado de Bielsa, quien de manera irónica refiere a los periodistas argumentos más cercanos a la persuasión que a la verdad.

El medio habilita nuevamente la voz de Bielsa:

\begin{abstract}
El Loco sí indagó más sobre la actualidad futbolística de Salford, como hace con todos los adversarios: "Salford utiliza un esquema posicional no tradicional, tiene algunas características del fútbol británico histórico. Defensas y delanteros altos, preparados para un tipo de fútbol que históricamente predominó en las Islas Británicas. También es un equipo moderno, ágil, dinámico. Es una buena mezcla de estilos" (La Nación, 2019, p. 2).
\end{abstract}

En este párrafo se representan características concretas de la metodología de Bielsa. El verbo indagó aclara la capacidad de análisis del entrenador, dejando de manifiesto dos cuestiones: el estudio y la previsión, ambos rasgos pertenecientes al raciocinio, que se articulan con un fútbol de laboratorio (Helal, 2000), planificado y mecánico.

Cuando describe al Salford, Bielsa menciona «algunas características del fútbol británico histórico» (La Nación, 2019, p.2). Archetti (2008) lo describe como un estilo monótono, mecánico, disciplinado y físico, esta última característica similar a la descrita por Bielsa (defensas y delanteros altos). Por otro lado, lo detalla como dinámico, descripción que en el fútbol actual se relaciona con las modificaciones paulatinas que la disciplina ha ido teniendo. En palabras de Álex Couto (2015), el fútbol se ha complicado porque ha incorporado la ciencia médica, la psicología, la nutrición, la tecnología informática y los cuantificadores de fuerza y resistencia. Estos recursos sumados a la progresión de tácticas y estrategias han complejizado el juego de manera dinámica y en simultáneo.

En el párrafo final, La Nación remarca de nuevo la estrategia enunciativa que ocupa el título de la nota:

Con cuatro puntos sobre seis posibles en el Championship (Sheffield Wednesday y Charlton son los únicos que triunfaron en las dos fechas), Leeds debuta en la Copa de la Liga ante un Salford que asume la rivalidad que le transmiten campeones de la época dorada del United (La Nación, 2019, p. 3).

El medio utiliza una metáfora (época dorada) para describir parte del legado histórico del Manchester United de Sir Álex Ferguson. Por otro lado, construye una rivalidad a partir de un

\footnotetext{
11 Platón presentó un pensamiento antirretórico principalmente contra los sofistas, reclamando que la retórica se apoyara en la filosofía para darle valor de verdad a la palabra. La consideración de la disciplina sofista como persuasión que trata de influir en los sentimientos y comportamiento de los hombres es su principal crítica. 
verbo que se utiliza como modalidad asertiva (asume), poniendo en común el intertexto histórico del enfrentamiento. La estrategia de La Nación, en ese caso, queda explícita en el título y en el final de la nota.

En la nota titulada «Los Pumas tienen su búnker en la casa embrujada: los fantasmas de Marcelo Bielsa en 2002» (La Nación, 2019, p. 1), la estrategia enunciativa utiliza el drama a partir de un enunciado apreciativo, el miedo, apelando a la fantasía (fantasmas) y a la magia negra (casa embrujada). La certeza del enunciado y su capacidad asertiva de enunciación construyen la figura de Bielsa a partir de la negatividad y de uno de los sucesos que esquematiza la construcción discursiva del bielsismo: el Mundial de Corea-Japón 2002. La Nación continúa desarrollando el tema:

La excursión albiceleste se extendió entre el 17 de mayo y el 14 de junio. Mucho menos de lo imaginado, porque la selección había llegado como candidata al título y ni atravesó la primera rueda. Entonces, nadie podía imaginar que tantos años después el complejo volvería a alojar a una selección argentina, ahora, a los Pumas en la antesala de un nuevo Mundial. Mucho menos, que se trataría de otro predio, aunque en el mismo lugar (La Nación, 2019, p. 2).

En este párrafo el medio da cuenta de lo sucedido en Corea-Japón 2002: Argentina era candidato y quedó eliminado en primera ronda. Esta certeza en el enunciado funciona como estructura discursiva fundamental para entender el bielsismo a partir de dos discursos performativos: la derrota en el Mundial y el imaginario del par victoria/derrota propio del nacionalismo deportivo. El legado deportivo nacional de la Selección Argentina también se pondrá en tensión con el resultado de Bielsa en aquel mundial. ${ }^{12}$

Pero la historia deportiva de Bielsa a partir de su experiencia como seleccionador argentino también tendrá la confrontación entre su discurso sobre el éxito y el nacionalismo deportivo. Dice Bielsa:

En la sociedad hay gente noble, franca, y también hay oportunistas. Cuando hay derrota, es de unos pocos; y cuando es victoria, es de todos. Ése es un principio que le hizo mucho daño al pueblo argentino. Porque nosotros no podemos perder todos juntos. Ganar sí, pero perder, siempre pierde alguien en particular, porque la derrota es vergonzosa y humillante. $\mathrm{Y}$ así está descrita, pero es mentira. Si hay algo que tiene valor

${ }^{12}$ Acorde a lo dicho en la página oficial de la Federación Internacional de Fútbol Asociada (FIFA), la Selección Argentina tiene 16 participaciones en la Copa del Mundo, de las cuales disputó 5 semifinales y 5 finales, ganando las de 1978 y 1986. En su continente, el equipo nacional cuenta con 14 Copas América. 
es no haber tenido una posición acomodaticia o demagógica frente a los episodios que me tocó vivir (lucht, 2011, p. 224).

La construcción del bielsismo por parte del medio obedece al espesor histórico que otorgan los triunfos en el fútbol, constitutivos de la esencia de los legados deportivos. Por otro lado, la derrota de Bielsa en Corea-Japón 2002 recupera la memoria nacional del «Desastre de Malmo ${ }^{13}$ porque en ambos mundiales la Selección Argentina era candidata a ganar el título. Las referencias al bielsismo a partir del Mundial del 2002 serán performativas y dialogarán con resultados y enunciados históricos del fútbol argentino.

Por otro lado, la nota «Habló Bielsa: cómo renueva la ilusión en Leeds a dos meses del fracaso de no ascender» (La Nación, 2019, p. 1), reaviva nuevamente la marca de Corea-Japón 2002. La palabra fracaso se articula rápidamente con una derrota deportiva (no ascender). El deíctico de tercera persona a partir de un verbo (habló Bielsa) detalla un acto de habla del entrenador, que adquiere importancia porque a partir de su nombre se organiza la enunciación. Luego el medio escribe: «Si bien Leeds, un histórico que intenta resurgir, había cumplido la mejor campaña en varios años, Bielsa estaba lejos de sentirse conforme» (La Nación, 2019, p. 1).

A través de dos enunciados lógicos (un histórico que intenta resurgir/ la mejor campaña en varios años), el medio da certeza del legado del Leeds y su articulación con la campaña de Bielsa. Si consideramos que el espesor de la historia deportiva depende de los triunfos, los últimos títulos del Leeds United ocurrieron en 1992, donde logró ganar la Liga de Fútbol de Primera División y la Community Shield (Leeds United Football Club, 2019). La estrategia enunciativa es dramatizada a partir de un verbo (resurgir), que carga de sentido el significado de histórico porque referencia el legado deportivo del Leeds. Luego el medio cita la palabra de Bielsa, que se convierte en locutor de la nota:

Tenemos la ilusión de mejorar lo de la temporada anterior. Nuestra obligación era ascender, no lo conseguimos y fue decepcionante para todos porque no cumplimos con las expectativas. Este año se renueva la posibilidad, ojalá estemos en condiciones de conseguir el ascenso (La Nación, 2019, p. 2).

13 En el año 1957, la Selección Argentina volvía a competir internacionalmente en el torneo Sudamericano, donde se proclamó campeón. Un año más tarde participó luego de 24 años en el Mundial de Suecia. En aquel evento, sufrió la eliminación en primera ronda por parte de Checoslovaquia, cayendo por 6 a 1. Aquel día fue denominado como «El desastre de Malmo». La no participación nacional en los mundiales de 1950 y 1954, la aparición de jugadores locales destacados en Europa y Colombia, y la Segunda Guerra Mundial, habían creado la autopercepción en el imaginario del fútbol argentino de que era el mejor del mundo. El segundo gobierno de Juan Domingo Perón colaboraba con el imaginario victorioso de «la edad de oro» del fútbol local, en donde no se participaba en el plano internacional para no perjudicar aquel discurso del éxito.

Question, Vol. 1, N. ${ }^{\circ}$ 65, abril 2020. ISSN 1669-6581

Instituto de Investigaciones en Comunicación | Facultad de Periodismo y Comunicación Social | Universidad Nacional de La Plata 
Bielsa es el locutor y a partir de la modalización en primera persona (tenemos) asume la responsabilidad de mejorar lo hecho en la primera temporada. El par utilizado por el entrenador (obligación/decepcionante) remarca la importancia que obtienen la victoria y la derrota en el deporte de competencia.

Si bien la importancia de ganar está implícita en el deporte de alto rendimiento, fue a partir de la década del '70 cuando se modificaron ciertos mecanismos del fútbol. Esto originó un crecimiento en la competitividad, una mayor seriedad en la participación del juego y una voracidad por conseguir victorias. Estos parámetros produjeron un juego más dinámico dentro del campo pero también fuera de él a través de sus discursos (Irungaray, 2019).

En la nota titulada "Championship. Leeds apenas empató: un gol polémico y el furor por el "MarceLego" Bielsa» (La Nación, 2019, p. 1), también aparecen esquematizaciones de los discursos sobre la victoria y la derrota. El enunciado apenas empató modaliza una enunciación asertiva, relacionado con lo insuficiente. En este caso, el empate significa no ganar y el medio construye su discurso a partir de reglas prescriptivas que ponen en valor la victoria en el fútbol nacional. Es por ello que el empate es escaso para el Leeds en la construcción de La Nación. Por otro lado, se hace referencia a una actitud enérgica (furor) por un muñeco réplica de Bielsa, que muestra directamente la relación entre el mercado y el fútbol. En el primer párrafo, La Nación recurre directamente a la palabra de Bielsa:

«Dos córners les permitieron a ellos meter un gol. Nosotros necesitamos diez chances para lo mismo» (La Nación, 2019, p. 1).

El locutor pone de manifiesto una alteridad de estilos. Por un lado un juego más directo, con pocas situaciones creadas y mucha efectividad; y por el otro un equipo que elabora más situaciones pero logra menos goles. Esto último caracteriza el estilo de Marcelo Bielsa, con equipos que como premisa proponen un fútbol ofensivo, donde las variables de ataque de un equipo son infinitas, en tanto que las defensivas son limitadas (Couto, 2015). La nota sigue:

"Creamos 10 situaciones de gol. Son suficientes para ganar el partido", añadió el Loco. Pero su equipo careció de puntería en el tramo final de la cancha. Pese a dominar la posesión y a tener controlado a su rival, sufrió un gol sobre el final que le costó el triunfo(La Nación, 2019, p. 3). La construcción del medio sobre la locura reaparece en este párrafo porque se identifica a Bielsa con esa caracterización. Sin embargo, también se le otorga nuevamente la voz al entrenador, que produce una descripción del estilo futbolístico del bielsismo. Luego, es el sujeto empírico el que arroja características del estilo del entrenador, destacando posesión y control sobre el rival, preceptos que fueron pregonados por Rinus Michels a fines de la década del '60 y principios del '70, primero en el Ajax de Ámsterdam y luego en la Selección de 
Holanda, apodada «La Naranja Mecánica». Sin embargo, el par (pese; sufrió) construye la estrategia enunciativa de manera negativa porque pone en primer plano la «no victoria» más allá del dominio sobre el rival. A su vez, el verbo sufrió le otorga drama a la actuación, una característica propia del campo del fútbol (Helal, 2000), que da cuenta sobre el empate del rival del Leeds sobre el final del partido. Luego La Nación concluye:

\begin{abstract}
En la previa del debut como local de Leeds se viralizaron fotos de muñecos de Lego con la cara y el cuerpo del Loco. La figura se completaba con los célebres binoculares del Spy-Gate y el banco individual que suele usar para sentarse mientras mira los partidos. El "MarceLego" Bielsa fue encargado por un shopping de Leeds para festejar el "Día de Yorkshire", que se celebró el pasado 1 de agosto. Las figuras pueden conseguirse en jugueterías de Leeds a un costo de 10,99 libras esterlinas (13,22 dólares estadounidenses). De hecho, tal como consignaron en redes sociales, muchos hinchas de Leeds llevaron su "MarceLego" al estadio. Una muestra más de que, a pesar de la decepción por no haber podido ascender a la Premier en la temporada pasada, la relación de idilio con el entrenador se mantiene intacta. (La Nación, 2019, p. 4).
\end{abstract}

En este párrafo aparece manifestada la noción de fútbol espectáculo, entendida como la mercantilización y mediatización del deporte. La figura de un Lego de Marcelo Bielsa refiere a la utilización de su figura por fuera del campo de juego con fines vinculados al marketing y la venta. La mención al Spygate ${ }^{14}$ y al asiento que utiliza Bielsa en los partidos (que se trata de un frigobar), forman parte de las particularidades mediáticas que construyen la figura del entrenador.

Por otro lado, hay un enunciado lógico que afirma una relación de idilio entre Bielsa y la hinchada de Leeds United, y que de manera consiguiente se articula en un romance con la ciudad entera. ${ }^{15}$ La palabra idilio le agrega épica al vínculo, lo cual entroniza la figura del entrenador, más allá de la carga negativa de la palabra decepción, argumentada por el no ascenso, traspolada en derrota. La estrategia enunciativa eleva a Bielsa por encima del resultado.

La nota «La última locura de Marcelo Bielsa: fue a la gala por el centenario de Leeds con el jogging de DT» (La Nación, 2019, p. 1), argumenta la estrategia enunciativa al principio del enunciado. La referencia a «la última locura» habilita el diálogo con otros enunciados y a su vez, se observan otras voces, otros enunciadores. El deíctico en tercera persona convierte la

${ }^{14}$ El capítulo 3 de la serie «Take us home: Leeds United» está dedicado al "Spygate", hecho por el cual un ayudante de Bielsa fue descubierto viendo un entrenamiento de su próximo rival, el Derby County. Posterior a la explosión mediática, el entrenador realizó una conferencia de prensa donde explicó que tenía datos de todos los equipos.

${ }^{15}$ En la serie «Take us home: Leeds United», se remarca que la ciudad entera es hincha del club, y lo caracterizan como un equipo popular de Inglaterra con una de las aficiones más apasionadas.

Question, Vol. 1, N. ${ }^{\circ}$ 65, abril 2020. ISSN 1669-6581

Instituto de Investigaciones en Comunicación | Facultad de Periodismo y Comunicación Social | Universidad Nacional de La Plata La Plata | Buenos Aires | Argentina

Página 13 de 20 
palabra última en una acción y el enunciado da certeza. La enunciación, sin embargo, refiere el acto como el último, poniendo en juego otras locuras anteriores.

Luego, el medio argumenta esa afirmación describiendo un acto particular de Bielsa. Esta particularidad reafirma las esquematizaciones del entrenador sobre las representaciones de la locura. Dentro de los discursos del fútbol espectáculo, estos esquemas funcionan a través de la mercantilización. La Nación utiliza esas características para trabajar la relación entre Bielsa y el entretenimiento que genera su persona. ${ }^{16}$ En el primer párrafo, el medio escribe:

\begin{abstract}
Marcelo Bielsa lo hizo de nuevo. El club, Leeds United, festejó su centenario en un acontecimiento con leyendas que se realizó en el propio estadio Elland Road. Hubo una cena a la que estuvieron invitados los integrantes del plantel profesional y el entrenador rosarino. El festejo era anunciado como un " evento de gala". Los presentes vistieron rigurosamente sacos, camisas, corbatas, moños, algún smoking. Un aspecto elegante y formal, como pedía el club. Todos, salvo uno (La Nación, 2019, p. 1).
\end{abstract}

La modalización en tercera persona articulada con un verbo (lo hizo de nuevo) remarca una recurrencia, articulada con la estrategia enunciativa del título. La singularidad de Bielsa de asistir a una fiesta de gala con la vestimenta deportiva del club es construida por La Nación a partir de la extrañeza y la peculiaridad. Lo extraño se articula con el imaginario de la locura porque es una acción que ocurre por fuera de la normalidad. Como explica lucht (2011), Bielsa se convirtió en un personaje, aunque a su pesar. El mercado del fútbol convierte este rasgo en una característica que construye discursivamente al bielsismo. Sobre el final de la nota, La Nación escribe:

\begin{abstract}
La ocurrencia no pasó inadvertida para algunos de los asistentes, que postearon fotos y videos en redes sociales. Lejos de criticar al entrenador argentino, los hinchas del equipo inglés que milita en el Championship y que el año pasado perdió el ascenso en las semifinales de los playoffs saludaron la forma que eligió Bielsa para concurrir a la celebración (La Nación, 2019, p. 2).
\end{abstract}

Se menciona nuevamente la relación de Bielsa con los hinchas del Leeds United. El medio destaca mediante un enunciado lógico que los hinchas "saludaron la forma que eligió Bielsa para concurrir a la celebración» (La Nación, 2019, p. 2). La estrategia enunciativa destaca una

\footnotetext{
${ }^{16}$ La revista El Gráfico trabajo esta relación entre el fútbol espectáculo y la figura de Bielsa cuando el entrenador se autodenuncio siendo técnico del Athletic de Bilbao. La locura en ese caso, conforma la figura de Bielsa como un rasgo mercantilista y de consumo.
}

Question, Vol. 1, N. ${ }^{\circ} 65$, abril 2020. ISSN 1669-6581

Instituto de Investigaciones en Comunicación | Facultad de Periodismo y Comunicación Social | Universidad Nacional de La Plata 
relación positiva de la afición del Leeds con el comportamiento del entrenador. ${ }^{17}$ La referencia al ascenso perdido se articula con los enunciados de la victoria y la derrota. La caracterización del comportamiento a partir de la palabra ocurrencia también deriva de las estructuras discursivas que construyen la locura en las referencias a Bielsa.

Finalmente, la nota «Marcelo Bielsa y el déja-vu de Leeds: ganaba 1-0, desperdició un penal y le empataron en el final» (La Nación, 2019, p. 1), marca la enunciación del título a partir de un deíctico de persona y una situación de recurrencia. El enunciado se presenta como lógico e indica una certeza: Bielsa no pudo ganar. Estos enunciados dialogan con la performatividad de los discursos nacionales sobre la victoria y la derrota por un lado, y con los discursos del legado deportivo del propio entrenador. El «deja-vu», en este caso, es una repetición cargada de negatividad porque, si bien indica un empate, la no victoria en la estrategia enunciativa de La Nación se construye de manera desfavorable. Luego el medio escribe:

\begin{abstract}
La misma historia. Leeds United, el equipo dirigido por Marcelo Bielsa, hizo todos los méritos para quedarse con la victoria frente a Derby County, pero falló en la puntada final y el rival encontró el empate 1-1 en el último instante. Es la tercera vez en la temporada que los dirigidos por el Loco dejan puntos jugando en Elland Road, su estadio. "Lo que nos ha pasado tantas veces, hoy fue peor que nunca. Recibimos un solo remate al arco y creamos entre 10 y 12 ocasiones", se lamentó el entrenador rosarino en la conferencia de prensa posterior al partido (La Nación, 2019, p. 1).
\end{abstract}

Hay una enunciación asertiva (la misma historia) que se articula con la estrategia enunciativa del título a partir de otros enunciadores polifónicos. Estas otras voces recuperan el imaginario deportivo de Corea-Japón 2002 porque presentan una situación similar: más allá de fabricar una cantidad de situaciones superior al rival, esto no decanta en victoria. Parte de la construcción del bielsismo se articula con este imaginario. La historia victoriosa de la Selección Nacional y el propio recorrido de Bielsa se apoyan sobre las narrativas del éxito, formando un imaginario sobre el entrenador. Las esquematizaciones discursivas obedecerán a aquel resultado en el Mundial de 2002. ${ }^{18}$

Por otro lado el medio utiliza la voz del entrenador, que a través de la deixis en primera persona (lo que nos ha pasado tantas veces) describe un comportamiento recurrente en el

\footnotetext{
${ }^{17}$ En la primera temporada de Bielsa en el Leeds, los hinchas recrearon la canción de la banda Queen «Bohemian Rhapsody» rebautizandola «Bielsa Rhapsody». En el capítulo 2 de «Take us home: Leeds United» se muestra como se pensó la idea.

${ }^{18}$ Como explica Román lucht, en Corea-Japón 2002, Argentina le ganó 1 a 0 a Nigeria, perdió por el mismo resultado ante Inglaterra, y empató 1 a 1 ante Suecia. En aquel torneo, la cantidad de situaciones de gol creadas por la Selección no pudo plasmarse en goles.

Question, Vol. 1, N. ${ }^{\circ}$ 65, abril 2020. ISSN 1669-6581

Instituto de Investigaciones en Comunicación | Facultad de Periodismo y Comunicación Social | Universidad Nacional de La Plata La Plata | Buenos Aires | Argentina

Página 15 de 20
} 
equipo. Los esquemas del estilo de los equipos de Bielsa son descritos por el entrenador: su equipo buscó atacar constantemente, en tanto el rival logró un solo remate al arco. El medio sigue citando la voz de Bielsa:

Nunca podemos pensar que recibir una sola chance de gol no es un mérito suficiente para ganar un partido. Lo concreto es que nos crearon una situación de gol en 90 minutos. $Y$ nosotros creamos $10 \ldots$ Si esa ecuación no alcanza para ganar...puntos suspensivos (La Nación, 2019, p. 2).

El entrenador continúa dando argumentos sobre su estilo futbolístico, el bielsismo. Este tipo de juego contiene características de otras escuelas del fútbol reconocidas, como el Fútbol Total y el estilo soviético de Valery Lobanovski, que plantearon ideas disruptivas en el juego. El Totaalvoetbal creado por Marinus Michels revolucionó los métodos de entrenamiento y la táctica, proponiendo un fútbol creativo e imaginativo combinado con el esfuerzo físico, la solidaridad y el compromiso colectivo. Por otro lado, fue Lobanovski el primero en solicitar ordenadores para verificar el funcionamiento de sus equipos a partir de su expertise como profesional de las matemáticas (Couto, 2015). Finalmente, y dentro del plano del fútbol argentino, el diálogo entre el bielsismo y los estilos de César Menotti y Carlos Bilardo, también forman parte de los discursos sobre Bielsa ${ }^{19}$.

La dualidad histórica entre menottismo y bilardismo forma parte del intertexto que dialoga con los discursos sobre Bielsa. El imaginario que identifica a Menotti con el estilo tradicional del fútbol local, y a Bilardo como un técnico de ideas europeas tendrá una incidencia de matriz sobre los discursos referentes al bielsismo. La histórica alteridad entre "la nuestra" y el estilo británico formarán esquemas y estereotipos sobre Bielsa. ${ }^{20}$ La relación del entrenador con algunas características del fútbol europeo las describe Couto (2015):

Todos los parámetros y variables que inciden en su trabajo son tenidos en cuenta, con un estudio que implica un enorme nivel intelectual y una capacidad para eliminar este sesgo, digna del mejor físico cuántico. Su modelo de entrenamiento incluye todos los parámetros tácticos existentes en el fútbol y los utiliza a su conveniencia, caracterizándose por ser un entrenador que a pesar de enfocar su filosofía del juego hacia la creación y el ataque, es capaz de manejar el proceso defensivo con mayor creatividad que nadie [...] (p. 37).

\footnotetext{
${ }^{19}$ Tanto Menotti como Bilardo adquieren pregnancia en el imaginario del fútbol argentino porque son los dos técnicos campeones del mundo con la Selección Argentina.

${ }^{20}$ Como afirma Eduardo Archetti (2017), es la revista El Gráfico la creadora de un estilo autóctono, al cual denomina "la nuestra", identificandolo con la gambeta, el desparpajo y el individualismo, características contrarias al fútbol europeo donde prima lo monótono, mecánico, la fuerza y el orden. 
El bielsismo desde los parámetros del fútbol es un mosaico de estilos. La Nación lo deja entrever y a su vez, pone a dialogar el contexto discursivo con los enunciados propios sobre Bielsa. El estilo del entrenador es analizable tanto desde los estilos performativos del fútbol nacional como de reconocidas escuelas europeas.

En el último párrafo, se produce la tensión discursiva entre Bielsa como locutor y el medio:

Sobre el futuro, el entrenador rosarino se preguntó: "¿Qué deberíamos cambiar? El juego consiste en atacar y defender. Si al atacar creamos diez veces más situaciones de las que crea el rival, no puede lamentar el procedimiento, el estilo". Y recalcó que Leeds "tendría que tener el $100 \%$ de los puntos". No es el caso: ya perdió 7 unidades como local. Una cifra que, al final de temporada, puede significar la diferencia entre ascender en forma directa o jugar los playoffs (La Nación, 2019, p. 3).

Nuevamente se habilita la voz de Bielsa, quien modaliza un enunciado lógico: «el juego consiste en atacar y defender» (La Nación, 2019, p. 3). Luego, a partir de un verbo, remarca la cantidad de puntos que debería tener el Leeds a partir de sus méritos. Este discurso se tensiona con el del medio, que pone en juego el imaginario de la victoria y la derrota. La descripción de lo que Bielsa entiende por el fútbol crea una disyuntiva con la importancia de la victoria que construye La Nación. La estrategia enunciativa del medio indica la importancia del deporte de competencia respecto a la victoria, y a su vez dialoga con los enunciados tradicionales del nacionalismo deportivo.

\section{Consideraciones finales}

A partir de este breve análisis es posible tomar en cuenta algunas consideraciones sobre La Nación y su construcción discursiva sobre Marcelo Bielsa. La primera de ellas es que la elección de los usos lingüísticos por parte del medio no son casuales, sino que responden a una relación estrecha con el contexto en donde son producidos. Es decir, enunciados actuales e históricos que dialogan con la producción enunciativa del medio.

Las similitudes y oposiciones que presenta el bielsismo como una forma de juego reconocida, y el diálogo con otros estilos del fútbol local e internacional, son enunciadas por el medio a partir de reglas prescriptivas y esquematizaciones discursivas. Las huellas dialógicas referidas al menottismo y al bilardismo, así como al Fútbol Total y el estilo soviético, se articulan con los enunciados que La Nación presenta a partir de la experiencia de Bielsa en el Leeds United. La enunciación de un bielsismo calculado y matemático, que analiza a los rivales e intenta de manera constante la minimización del error, serán enunciados articulados con los esquemas que el medio construye.

Question, Vol. 1, N. ${ }^{\circ}$ 65, abril 2020. ISSN 1669-6581

Instituto de Investigaciones en Comunicación | Facultad de Periodismo y Comunicación Social | Universidad Nacional de La Plata 
Tanto el deporte como espectáculo a partir de lo ocurrido dentro del campo de juego como el negocio generado por fuera de él, también son variables que caracterizan la complejidad del fútbol. Las particularidades que presenta la figura de Bielsa con sus comportamientos o su vestimenta forman un personaje posible de ser vendido a partir de su mediatización y posteriormente, de su mercantilización. Es por ello que el bielsismo es estilo de juego, pero también mediatización y mercancía.

Por otro lado, la experiencia actual de Bielsa como técnico en Leeds constantemente se articula con la importancia de la victoria, que funciona intertextualmente como punto de origen de los discursos del fútbol argentino y dialoga en simultáneo con su legado deportivo. Por otra parte, el discurso del entrenador respecto a la victoria y la derrota se tensiona con el relato del medio, pero también con la lógica triunfalista del relato nacional y el imaginario del fútbol.

Finalmente, fue posible dar cuenta de reglas prescriptivas y esquemas discursivos que especifican regularidades sobre Bielsa: la caracterización de loco, el estudio y el raciocinio aplicado al fútbol, el Mundial de Corea-Japón 2002 y su relación con el imaginario europeo. Todos estos discursos dialogan simultáneamente con las construcciones discursivas que La Nación hace sobre el bielsismo. Sin embargo, las caracterizaciones no solamente obedecen a la disciplina del fútbol, sino que se inmiscuyen en discursos por fuera del campo de juego que refieren al entrenador. El bielsismo se torna entonces un discurso con complejidades, pero compuesto de regularidades a partir de la construcción del medio.

\section{Referencias bibliografícas}

Alabarces, P. (2002). Fútbol y patria. El fútbol y las narrativas de la nación en Argentina. Buenos Aires, Argentina: Prometeo.

Angenot, M. (2010). El discurso social: los límites históricos de lo pensable y lo decible. Buenos Aires, Argentina: Siglo XXI Editores.

Archetti, E. (2008). El potrero y el pibe. Territorio y pertenencia en el imaginario del fútbol argentino. Horizontes antropológicos (30), 259-282.

Archetti, E. (2017). Estilo y virtudes masculinas en El Gráfico: la creación del imaginario del fútbol argentino. En Eduardo Archetti: antología esencial (pp. 461-497). Ciudad Autónoma de Buenos Aires, Argentina: CLACSO.

Bajtín, M. (1982). El problema de los géneros discursivos. Estética de la creación verbal (pp. 248-293). México: Siglo XXI. 
Championship. Leeds apenas empató: un gol polémico y el furor por el "MarceLego" Bielsa (10 de agosto de 2019). La Nación. Recuperado de https://www.lanacion.com.ar/deportes/leeds-empato-lego-bielsa-bronca-arbitro-faltanid2276221

Couto, A. (2013). Las grandes escuelas del fútbol moderno. Fútbol de Libro.

Federación Internacional de Fútbol Asociado (FIFA). (2019). Historia mundialista. Recuperado de https://bit.ly/2R4ZLCa

Foucault, M. (1964). Historia de la locura en la época clásica (Trad.Juan José Utrilla). Santa Fé de Bogotá, Colombia: Fondo de Cultura Económica.

García García, F. (2005). Una aproximación a la historia de la retórica. Revista de comunicación y tecnologías emergentes, (2). Recuperado de http://bit.ly/32MkuRo

Grosso, C. (16 de septiembre de 2019). Los Pumas tienen su búnker en la casa embrujada: los fantasmas de Marcelo Bielsa en 2002. La Nación. Recuperado de https://www.lanacion.com.ar/deportes/rugby/los-pumas-casa-embrujada-fantasmasmarcelo-bielsa-nid2287820

Habló Bielsa: cómo renueva la ilusión en Leeds a dos meses del fracaso de no ascender (20 de julio de 2019). La Nación. Recuperado de https://www.lanacion.com.ar/deportes/bielsarenueva-ilusion-leeds-dos-meses-despues-nid2269661

Helal, R. (2000). As Idealizações de Sucesso no Imaginário Futebolístico Brasileiro: um estudo de caso. Peligro de Gol. Estudios sobre deporte y sociedad en América Latina (pp. 101-111). Buenos Aires: CLACSO.

Hicken, L. (Director). (2019). Take us home: Leeds United (Serie). Recuperado de https://www.pelisplay.tv/serie/take-us-home-leeds-united/temporada-1

Irungaray, G. (2019). El Gráfico: épica, potrero y bielsismo en el relato del fútbol argentino (Tesis de grado). Recuperado de http://bit.ly/32QMmUi

lucht, R. (2010). La vida por el fútbol. Marcelo Bielsa, el último romántico. Recuperado de http://bit.ly/2KQfgOz

La última locura de Marcelo Bielsa: fue a la gala por el centenario de Leeds con el jogging de DT (18 de octubre de 2019). La Nación. Recuperado de https://www.lanacion.com.ar/deportes/futbol/la-ultima-locura-marcelo-bielsa-fue-gala$\underline{\text { nid2298210 }}$

Leeds United Football Club. (2019). Club honours. Recuperado de http://bit.ly/2K29Ejk

Maingueneau, D. (2009). Enunciado y contexto. Análisis de textos de comunicación (pp. 9-19). Buenos Aires: Nueva Visión. 
Manchester United FC. (2019). The story of our rivalry with Leeds. Recuperado de http://bit.ly/2PVIUoL

Marcelo Bielsa y el déja-vu de Leeds: ganaba 1-0, desperdició un penal y le empataron en el final (21 de septiembre de 2019). La Nación. Recuperado de https://www.lanacion.com.ar/deportes/futbol/bielsa-deja-vu-de-leeds-volvio-perderpuntos-nid2290049

Mauri, C. (12 de agosto de 2019). Bielsa y un clásico impensado por obra de viejas glorias de Manchester United. La Nación. Recuperado de https://www.lanacion.com.ar/deportes/futbol/bielsa-nid2276848

Moyano, M. (2008). Literatura, Estado y Nación en el siglo XIX argentino: el poder instituyente del discurso y la configuración de los mitos fundacionales de la identidad. Amérique Latine Histoire et Mémoire, Les Cahiers ALHIM. Recuperado de https://journals.openedition.org/alhim/2892

Puig, L. (2004). Polifonía lingüística y polifonía narrativa. Acta poética. Recuperado de http://bit.ly/2KnKAUd

Secul Giusti, C. (2016). Rompiendo el silencio: la construcción discursiva de las líricas de rockpop argentino durante el período 1982-1989 (Tesis de Doctorado). Recuperado de http://bit.ly/2zfKE2s

Tusón, A. y Calsamiglia, H. (2001). Las cosas del decir. Manual de análisis del discurso. Barcelona, España: Editorial Ariel.

Volóshinov, V. N. (2009). El marxismo y la filosofía del lenguaje (Trad. Tatiana Bubnova). Buenos Aires, Argentina: Ediciones Godot. 